\title{
An Interactive Dialogue Modelling Editor for Designing Multimodal Applications
}

\author{
Sebastian Feuerstack \\ Universidade Federal de São Carlos, \\ Departamento de Computacão, \\ Rod. Washington Luis, km 235 - SP, Brazil \\ +49307762324 \\ sfeu@cs.tu-berlin.de
}

\begin{abstract}
This poster presents initial results of an approach for (1) designing and implementing a dialogue modelling editor to enable the design of multimodal applications, that (2) supports the design for various multimodal setups and that (3) is specifically targeted to be used by interaction designers.
\end{abstract}

\section{Categories and Subject Descriptors}

H.5.2 [Information Interfaces and Presentation]: User Interfaces - Input devices and strategies, Interaction styles, Prototyping; D.2.2 [Software Engineering]: Design Tools and Techniques - User Interfaces.

\section{General Terms}

Design, Human Factors, Languages.

\section{Keywords}

Model-based User Interface Development, Multimodal Interfaces, Software Engineering, Interactive Editor, HCI.

\section{INTRODUCTION}

Nowadays multimodal systems that support the user by a combination of speech, gesture and graphical-driven interaction are already part of our everyday life. Examples are combinations of speech-driven and graphical interfaces like in-car assistance systems, language-learning software, or tourist information systems. The market success of recent video-games that can be controlled in a more natural and intuitive way by using hand gestures, balancing and moving the body demonstrates that even new audiences can be addressed by enabling multimodal interaction to ease the usage of interactive systems.

\section{CHALLENGES}

Modelling multimodal systems that support various multimodal setups is an open research issue. A recent promising work by [11]

Permission to make digital or hard copies of all or part of this work for personal or classroom use is granted without fee provided that copies are not made or distributed for profit or commercial advantage and that copies bear this notice and the full citation on the first page. To copy otherwise, or republish, to post on servers or to redistribute to lists, requires prior specific permission and/or a fee.

SIGDOC 2010, September 2729, 2010, S.Carlos, SP, Brazil. Copyright 2010 ACM 978-1-4503-0403-0...\$5.00. that implemented a model-based development process to generate multimodal web interfaces stated the importance of considering the CARE-properties, but neglected the support of modelling complementary or redundant multimodal interaction to support multimodal fusion. UsiXML [6] and TERESA [2] do not offer a dialogue model but distribute it to several models (mainly the task model that specifies the interaction sequence and the abstract user interface model containing the navigation). Since these models are considered modality independent, supporting different navigations based on the used combination of modalities is difficult to implement. Research how to design multimodal interfaces has resulted in both modality-independent and modality-dependent dialogue models. The latter ones concentrate on proposing dialog models to design a certain modality (mainly graphics like $[12,8]$ or a specific multimodal setup [11]. The former ones propose a set of abstractions by interactors or generic widgets that are transformed by rules $[6,2]$ or interpreted at runtime to result in concrete widgets [10].

In practice, the distinction between abstract dialogue and concrete interaction isn't as clear cut. The main problem is that the structure of interactors will often change when one takes the interaction technique into account. A voice application is often more sequential, interfaces for small devices are often more modal, a direct manipulation application is difficult to split into smaller parts, since actions are projected into the graphical objects. So, even though the dialog is abstract and do not explicitly refer to interaction technique, the interaction technique affects it in (sometimes subtle) ways. Thus it keeps questionable if these modalities have more in common than that they differ in order to argument for a modality independent dialogue model.

\section{DIALOGUE EDITOR}

We are focusing our work on realizing an interactive editor for designing multimodal applications that support all four relations of combining the modes of a multimodal system: Complementary, Assignment, Redundancy, and Equivalence (the CARE properties) [7].

The editor allows visual programming of a dialogue model by interaction designers and connects with the help of an interpreter that can execute these dialogue models to the Open Interface Framework, which implements a standardized framework and a component repository to integrate several interaction devices and sensors into one unique platform that enables a developer to flexibility combine and aggregate various devices and sensors to form multimodal interaction setups [5]. 
The dialogue design consists of two basic steps: First, profiles are described that characterize a multimodal interaction setup which we understand as a set of interconnected Open Interface Components. The characterization is based on a fine-grained taxonomy of output modalities from [13] and the combination space from [1] that enables the definition of composite modalities. The profiles are implemented by using the Pipelines-Model of Open Interface.

Second, we design for one profile a dialogue model. For every further profile, the dialog editor generates a draft dialogue design based on a comparison of the original profile to the new profile. These concrete dialogue designs can be run by an interpreter that executes the dialogue flow and calls or reacts on the Open Interface Components and the application.

By using the Open Interface Platform we can benefit from a lot of already existing fusion and signal processing components as well as device drivers to keep the focus on the dialogue modelling editor that will consider and merge previous editors that offer dialogue model but does not support multimodal design.

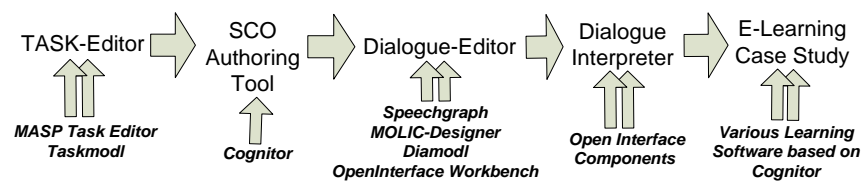

Figure 1: The principle tool-chain to design multimodal dialogues for the case studies.

The implementation of the editor will be based on directly extending and merging relevant parts and suitable notations of previous editors for dialogue modelling to support multimodal modelling: Therefore we consider the Speechgraph editor, the Diamodl editor [12] as well as the MoLIC designer [9] and the Dialogue Graphs approach [8] (see figure 1).

\section{APPLICATION IN E-LEARNING}

Multimodal approaches to learning have been proven to be extremely effective since information introduced aurally, visually and kinaesthetically can significantly increase the possibility of understand and remembering information [4].

The evaluation focuses on the suitability and efficiency of the dialogue modelling editor and will be performed by following an interdisciplinary approach involving both, interaction designers and domain experts that will design a multimodal teaching application based by applying the dialog modelling notation.

Figure 1 illustrates the principle tool chain that should be supported to generate dialogues describing multimodal interaction. The first row of figure 1 presents the final tools, the second row already existing tools that are considered for extension. For the case studies the project will use an already existing SCORM authoring tool, the Cognitor [3].

\section{ACKNOWLEDGMENTS}

The author is grateful to the Deutsche Forschungsgemeinschaft (DFG) for the financial support of his work.

\section{REFERENCES}

[1] N. O. Bernsen. Modality theory in support of multimodal interface design. In Proceedings of the AAAI Spring Symposium on Intelligent Multi-Media Multi-Modal Systems, pages 37-44, 1994.

[2] Silvia Berti, Francesco Correani, Giulio Mori, Fabio Paterno, and Carmen Santoro. TERESA: A transformationbased environment for designing and developing multidevice interfaces. In ACM CHI 2004, Extended Abstract, volume II, pages 793-794, Vienna, Austria, April 2004. ACM Press.

[3] David Buzatto, Junia Coutinho Anacleto, and Ana Luiza Dias. Providing culturally contextualized metadata to promote sharing and reuse of learning objects. In SIGDOC '09: Proceedings of the 27th ACM international conference on Design of communication, pages 163-170, New York, NY, USA, 2009. ACM.

[4] Alice M. Hammel. Using multi-modal techniques to motivate intuitive and non-intuitive students. American Music Teacher, Oct-Nov., 2003.

[5] Jean-Yves Lionel Lawson, Ahmad-Amr Al-Akkad, Jean Vanderdonckt, and Benoit Macq. An open source workbench for prototyping multimodal interactions based on off-the-shelf heterogeneous components. In EICS '09: Proceedings of the 1st ACM SIGCHI symposium on Engineering interactive computing systems, pages 245-254, New York, NY, USA, 2009. ACM.

[6] Quentin Limbourg, Jean Vanderdonckt, Benjamin Michotte, Laurent Bouillon, and Victor Lopez-Jaquero. USIXML: A language supporting multi-path development of user interfaces. In Remi Bastide, Philippe A. Palanque, and Joerg Roth, editors, EHCI/DS-VIS, volume 3425 of Lecture Notes in Computer Science, pages 200-220. Springer, 2004.

[7] Laurence Nigay and Joelle Coutaz. Multifeature systems: The care properties and their impact on software design. In Intelligence and Multimodality in Multimedia Interfaces. 1997.

[8] Daniel Reichard, Peter Forbrig, and Anke Dittmar. Task models as basis for requirements engineering and software execution. In Proceedings of TAMODIA 2004, pages 51 57, Prague, Czeck Republic, 2004. ACM Press.

[9] Ugo Braga Sangiorgi and Simone D.J. Barbosa. Molic designer: towards computational support to hci design with molic. In EICS '09: Proceedings of the 1st ACM SIGCHI symposium on Engineering interactive computing systems, pages 303-308, New York, NY, USA, 2009. ACM.

[10] Robbie Schaefer. Model-based Developent of Multimodal and Multi-Device User Interfaces in Context-Aware Environments. PhD thesis, C-LAB Publication, Band 25, Shaker Verlag, Aachen, 2007.

[11] Adrian Stanciulescu. A Methodology for Developing Multimodal User Interfaces of Information Systems. $\mathrm{PhD}$ thesis, Universite Catholique de Louvain, 2008.

[12] Hallvard Traetteberg. Dialog modelling with interactors and uml statecharts - a hybrid approach. In DSV-IS, pages 346361, 2003.

[13] Frederic Vernier and Laurence Nigay. A framework for the combination and characterization of output modalities. Lecture Notes in Computer Science, 1946:35ff, 200 